\title{
Amorphous Cr-Ti Texture-inducing Layer Underlying (002) Textured bec-Cr alloy Seed Layer for FePt-C Based Heat-assisted Magnetic Recording Media
}

\author{
Seong-Jae Jeon ${ }^{1 *}$, Shintaro Hinata ${ }^{1,2}$, and Shin Saito ${ }^{1}$ \\ ${ }^{1}$ Department of Electronic Engineering, Tohoku University, 6-6-05 Aoba, Aramaki, Aoba-ku, Sendai 980-8579, Japan \\ ${ }^{2} J a p a n$ Society for the Promotion of Science Research Fellow (PD), Tohoku University, 6-6-05 Aoba, Aramaki, Aoba-ku, \\ Sendai 980-8579, Japan
}

(Received 21 November 2015, Received in final form 19 February 2016, Accepted 14 March 2016)

\begin{abstract}
$\mathrm{Cr}_{100-\mathrm{x}} \mathrm{Ti}_{\mathrm{x}}$ amorphous texture-inducing layers (TIL) were investigated to realize highly (002) oriented $\mathrm{L1}_{0} \mathrm{FePt}$ $\mathrm{C}$ granular films through hetero-epitaxial growth on the (002) textured bcc-Cr $\mathbf{H}_{80} \mathrm{Mn}_{20}$ seed layer (bcc-SL). Asdeposited TILs showed the amorphous phase in Ti content of $30 \leq x($ at $\%) \leq 75$. Particularly, films with $40 \leq x$ $\leq 60$ kept the amorphous phase against the heat treatment over $600{ }^{\circ} \mathrm{C}$. It was found that preference of the crystallographic texture for bec-SLs is directly affected by the structural phase of TILs. (002) crystallographic texture was realized in bcc-SLs deposited on the amorphous TILs $(40 \leq x \leq 70)$, whereas (110) texture was formed in bec-SLs overlying on crystalline TILs $(x<30$ and $x>70)$. Correlation between the angular distribution of (002) crystal orientation of bec-SL evaluated by full width at half maximum of (002) diffraction (FWHM) and a grain diameter of bec-SL indicated that while the development of the lateral growth for bec-SL

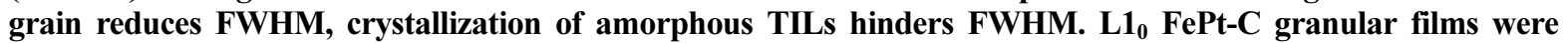
fabricated under the substrate heating process over $600{ }^{\circ} \mathrm{C}$ with having different FWHM of bcc-SL. Hysteresis loops showed that squareness $\left(M_{\mathrm{r}} / M_{\mathrm{s}}\right)$ of the films increased from 0.87 to 0.95 when FWHM of bcc-SL decreased from $13.7^{\circ}$ to $3.8^{\circ}$. It is suggested that the reduction of (002) FWHM affects to the overlying MgO film as well as FePt-C granular film by means of the hetero-epitaxial growth.
\end{abstract}

Keywords : amorphous Cr-Ti texture inducing layer, bcc-Cr alloy seed layer, lateral grain growth, hetero-epitaxial growth, $\mathrm{L}_{0}$ FePt-C granular film, angular distribution of $c$-axis

\section{Introduction}

The hard disk drive (HDD) industry has been gradually developed over several decades by increasing the areal density (AD). While current HDD based on CoPtCr-oxide granular film is expected to limit the maximum $\mathrm{AD}$ of 1.0-1.5 Tb/in ${ }^{2}$, heat-assisted magnetic recording (HAMR) media based on $\mathrm{L} 1_{0} \mathrm{FePt}$ granular film is considered to extend the maximum $\mathrm{AD}$ over $4 \mathrm{~Tb} / \mathrm{in}^{2}$ owing to the high magnetocrystalline anisotropy of $\mathrm{L}_{0}$ ordered phase for FePt $\left(K_{\mathrm{u}} \sim 7 \times 10^{7} \mathrm{erg} / \mathrm{cm}^{3}\right)$ [1-3]. In order to realize the $\mathrm{L}_{0}$ ordered $\mathrm{FePt}$ granular film as a high recording density medium, it is necessary to enhance the signal-tonoise ratio (SNR). Contribution to the SNR can result from variation in the angular distribution of $c$-axis orientation of $\mathrm{L} 1_{0} \mathrm{FePt}$ grains. Since the $c$-axis orientation,

CThe Korean Magnetics Society. All rights reserved.

*Corresponding author: Tel: +81-80-3191-1307

Fax: +81-22-263-9402, e-mail: jsjigst@ecei.tohoku.ac.jp which corresponds to the easy magnetized axis for the $\mathrm{L} 1_{0}$ ordered $\mathrm{FePt}$, can be adjusted by hetero-epitaxial growth from (002) textured crystalline seed layer (SL), the angular distribution of the $c$-axis is mainly attributed to that of SL. Recently, we reported the concept to suppress the angular distribution of the (002) orientation of SL [4]. The origin of the angular distribution of the crystal orientation can be described by considering the formation of crystalline facets during the solidification of the liquid phase of sputtered atoms under the different wettability of SL on an amorphous texture-inducing layer ( $a$-TIL). Low wettability condition induces the ballshaped liquid phase of the sputtered atoms, whereas high wettability condition tends to spread the liquid phase over the surface of $a$-TIL. During the solidification, the liquid phase changes to the crystalline facet in order to form the crystallographic texture. In the case of the low wettability, the crystalline facets have much more chance to form the crystalline plane with various angles from normal to the film plane. Consequently, the crystallographic texture can 


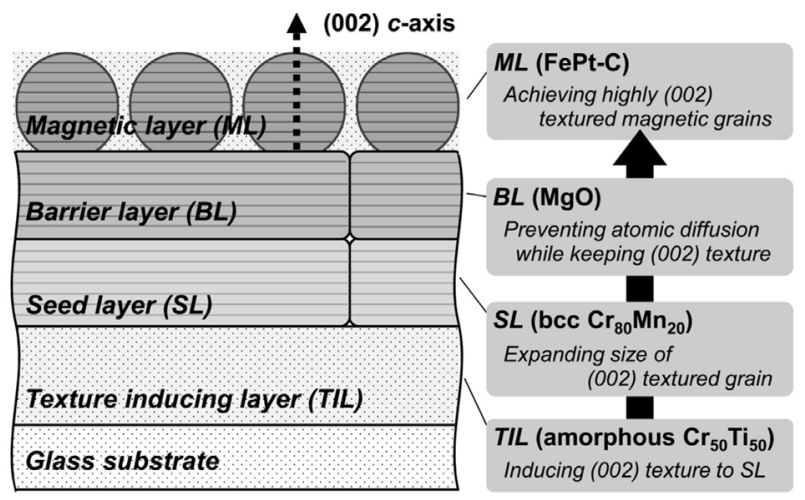

Fig. 1. Schematic representation to suppress angular distribution of (002) $c$-axis orientation for FePt-C granular film.

be slanted from normal to the film plane. In the case of the high wettability, the most surface of the crystalline facets is parallel to the normal to the plane, which induces the development of the crystallographic texture without a tilt of the crystal orientation. Providing that the solidification determines the texture, fabrication of the large grain SL is essential. Based on the concept, a designed stacking structure is illustrated in Fig. 1. We found that the materials combination between bcc-Cr alloy SL and $\mathrm{Cr}_{50} \mathrm{Ti}_{50} a$-TIL promoted the lateral grain growth for bcc$\mathrm{Cr}$ alloy SL. However, crystallization of the $\mathrm{Cr}_{50} \mathrm{Ti}_{50} a$ TIL induced by post-annealing process suppressed the development of the lateral grain growth. In order to increase the grain size of bcc-Cr alloy SL, investigation on the degree of amorphous and thermal stability of amorphous is inevitable. In this research, quantitative study on the amorphous Cr-Ti TILs was carried out for (002) texture formation of bec-Cr alloy SL.

\section{Experimental Procedure}

All samples used in this study were fabricated by using in-line magnetron sputtering system (Canon Anelva C3010). The stacking structure of the samples was composed of $\mathrm{Cr}_{80} \mathrm{Mn}_{20}(30 \mathrm{~nm}) / \mathrm{Cr}_{100-\mathrm{x}} \mathrm{Ti}_{\mathrm{x}}(20 \mathrm{~nm}) / \mathrm{Ni}_{60} \mathrm{Ta}_{40}(2 \mathrm{~nm}) /$ nanocrystalline glass substrate. Here, $\mathrm{Ni}_{60} \mathrm{Ta}_{40}$ was introduced to promote the amorphous structure for $\mathrm{Cr}_{100-\mathrm{x}} \mathrm{Ti}_{\mathrm{x}}$ TIL. $\mathrm{Cr}_{100-\mathrm{x}} \mathrm{Ti}_{\mathrm{x}}$ TIL was deposited by co-sputtering $\mathrm{Cr}$ and Ti under $0.6 \mathrm{~Pa}$ Ar with a deposition rate of $0.5 \mathrm{~nm} / \mathrm{sec}$. Ti content was varied from 0 to $100 \mathrm{at} \%$, and then $\mathrm{Cr}_{80} \mathrm{Mn}_{20}$ was deposited on $\mathrm{Cr}_{100-\mathrm{x}} \mathrm{Ti}_{\mathrm{x}}$ TIL under $0.8 \mathrm{~Pa} \mathrm{Ar}$ with a deposition rate of $3.2 \mathrm{~nm} / \mathrm{sec}$. The substrate temperature could be elevated from RT to $600{ }^{\circ} \mathrm{C}$ before deposition of bcc- $\mathrm{Cr}_{80} \mathrm{Mn}_{20}$ SL (Hereafter, bcc-SL) to induce the lateral growth of bcc-SL grains without the atomic diffusion due to the phase stability of the alloy system [5]. The base pressure of all chambers was kept approximately $5 \times 10^{-6} \mathrm{~Pa}$. Structure analysis was carried out by X-ray diffractometry (XRD). The grain size of bcc-SL was derived by using Scherrer's equation with a full width at half maximum (FWHM) at a Bragg angle for CrMn (200) diffraction obtained from in-plane XRD [6]. The angular distribution of (002) crystal orientation of bcc-SL was evaluated by measuring rocking curve at (002) diffraction. Magnetic properties of FePt-C granular films were investigated by a SQUIDs-VSM with an applied magnetic field normal to the film plane up to 65 $\mathrm{kOe}$ at room temperature.

\section{Result and Discussion}

\subsection{Amorphous formation and its thermal stability of} $\mathrm{Cr}_{100-\mathrm{x}} \mathrm{Ti}_{\mathrm{x}}$ TILs

Figure 2 shows in-plane XRD patterns of (a) asdeposited and (b) post-annealed up to a temperature of $600{ }^{\circ} \mathrm{C}$ for $\mathrm{Cr}_{100-\mathrm{x}} \mathrm{Ti}_{\mathrm{x}}(20 \mathrm{~nm})$ TILs on $\mathrm{NiTa}(2 \mathrm{~nm}) /$ glass substrate with various $\mathrm{Ti}$ content. In the case of (a) asdeposited state, two diffractions at Bragg angles of $2 \theta_{\chi}=$ $44^{\circ}$ and $64^{\circ}$ were observed in $x=0$ (pure bcc-Cr) TIL. These diffractions, which were identified (110) and (220) diffractions of bcc-Cr powder, gradually shifted to lower angles with the increase of $x=0$ to $20 \mathrm{at} \%$. The result indicates that the atomic substitution from $\mathrm{Cr}$ to $\mathrm{Ti}$ induces the increase of the lattice volume while sustaining the crystalline structure. However, change of the diffraction width from the narrow to broad signal was observed with the further increase of $x$ from 20 to 30 at $\%$, and then the broad signal was obtained regardless of the increase of $x$
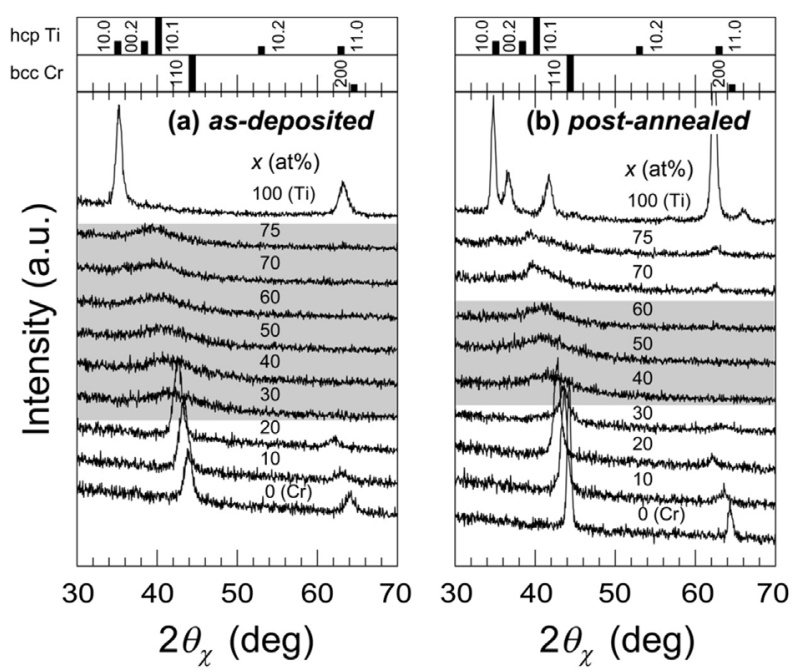

Fig. 2. In-plane XRD patterns of (a) as-deposited and (b) postannealed $\mathrm{Cr}_{100-\mathrm{x}} \mathrm{Ti}_{\mathrm{x}}(20 \mathrm{~nm})$ TILs on $\mathrm{NiTa}(2 \mathrm{~nm}) /$ glass substrate with various Ti content $(x)$. 
up to 75 at $\%$. This result suggests that the amount of the constructive interference of X-rays decreased due to the randomly located atoms (i.e. amorphous phase). Again, two diffractions at Bragg angles of $2 \theta_{\chi}=35^{\circ}$ and $63^{\circ}$ were obtained in $x=100$ (pure hcp-Ti) TIL. In the case of (b) post-annealed TILs, one can see that additional diffractions occurred at $2 \theta_{\chi}=40-44^{\circ}$ and $64^{\circ}$ in $x=30$, 70 , and 75 at $\%$ as compared with the as-deposited TILs. The result suggests that these films experienced the crystallization. Only the films with $x$ from 40 to 60 at $\%$ maintained the amorphous phase against the heat treatment.

\subsection{Preferred crystal orientation and its angular dis- tribution of bec-SL on $\mathrm{Cr}_{100-\mathrm{x}} \mathrm{Ti}_{\mathrm{x}}$ TILs}

Figure 3 shows out-of-plane XRD patterns of bcc-SL on $\mathrm{Cr}_{100-\mathrm{x}} \mathrm{Ti}_{\mathrm{x}}(20 \mathrm{~nm}) / \mathrm{NiTa}(2 \mathrm{~nm}) /$ glass substrate with various $x$ (a) without and (b) with the substrate heating process. In the case of (a) bcc-SL without substrate heating process, a continuous change in preference of the crystallographic texture was observed with the increase of $x$. Distinct diffraction at $2 \theta \sim 44^{\circ}$ was observed when bcc-SL was deposited on pure $\mathrm{Cr}(x=0)$ TIL. It was confirmed that the (110) sheet texture was formed in bccSL by comparing Bragg angle of bcc-SL to that of bcc-Cr powder. The intensity of the (110) diffraction rapidly decreased with the increase of $x$ from 0 to 30 at $\%$, and then the preference of the crystallographic texture was vanished at $x=40$ at $\%$. However, change in the preferred crystal orientation from (110) to (002) was observed at $x$ $=50$ and 60 at $\%$. Further increase of $x$ induced (110) sheet texture for bcc-SL. In the case of (b) bcc-SL with the substrate heating process, integrated intensity of all
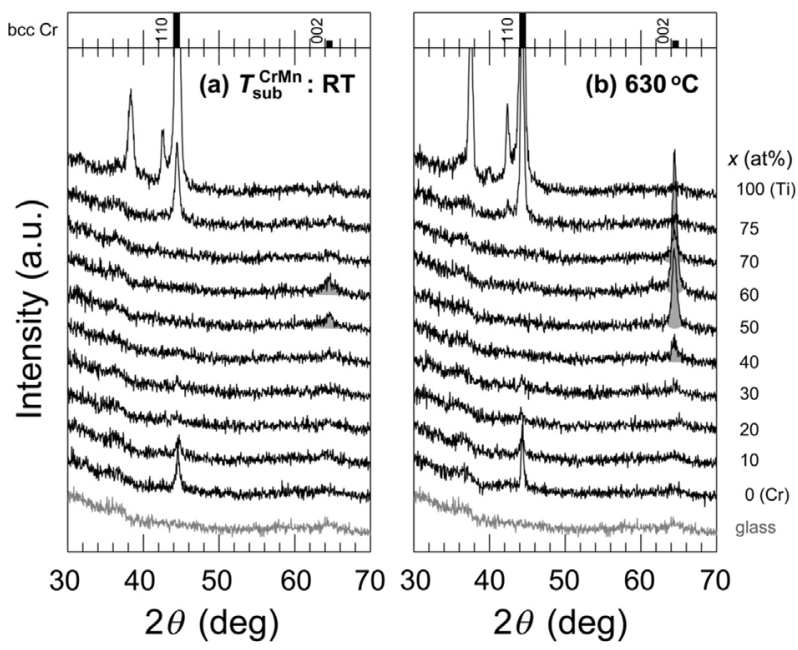

Fig. 3. Out-of-plane XRD patterns of bcc-SL on $\mathrm{Cr}_{100-\mathrm{x}} \mathrm{Ti}_{\mathrm{x}}(20$ $\mathrm{nm}) / \mathrm{NiTa}(2 \mathrm{~nm}) /$ glass substrate with various $\mathrm{Ti}$ content (a) without and (b) with substrate heating process.

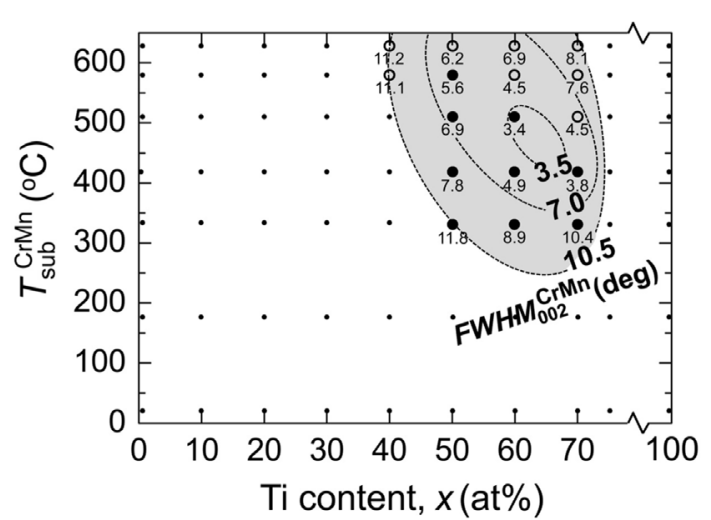

Fig. 4. Angular distribution of (002) crystal orientation (FWHM) of bcc-SL varied with substrate temperature $\left(T_{\text {sub }}\right)$ and Ti content $(x)$.

diffractions increased. Furthermore, one can see that the extra (002) diffraction appeared at $x=40$ and 70. It is suggested that the increased grain size enhanced the amount of constructive interference of X-ray. It is worth noting that (002) texture formation in bcc-SL is closely related to the formation of amorphous phase in $\mathrm{Cr}_{100-\mathrm{x}} \mathrm{Ti}_{\mathrm{x}}$ TILs.

Figure 4 shows the angular distribution of (002) crystal orientation of bcc-SL derived from the full width at half maximum (FWHM) varied with $T_{\text {sub }}$ and $x$. Measured range for (002) sheet texture (gray region) is limited by both $T_{\text {sub }} \geq 300{ }^{\circ} \mathrm{C}$ and $30<x($ at $\%)<75$. Note that this composition range corresponds to the amorphous formation region for $\mathrm{Cr}_{100-\mathrm{x}} \mathrm{Ti}_{\mathrm{x}}$ TILs. In addition, the magnitude of FWHM along the same $x$ decreased with increasing $T_{\text {sub }}$ (solid circle $\bullet$ ) and then, decreased with further increas$\operatorname{ing} T_{\text {sub }}$ (open circle $\circ$ ) within $x$ of 50 to 70 at $\%$.

Figure 5 shows variation in grain diameter (GD) of bccSL varied with $T_{\text {sub }}$ and $x$. To evaluate GD, CrMn (200) peak measured by in-plane X-ray diffraction was used

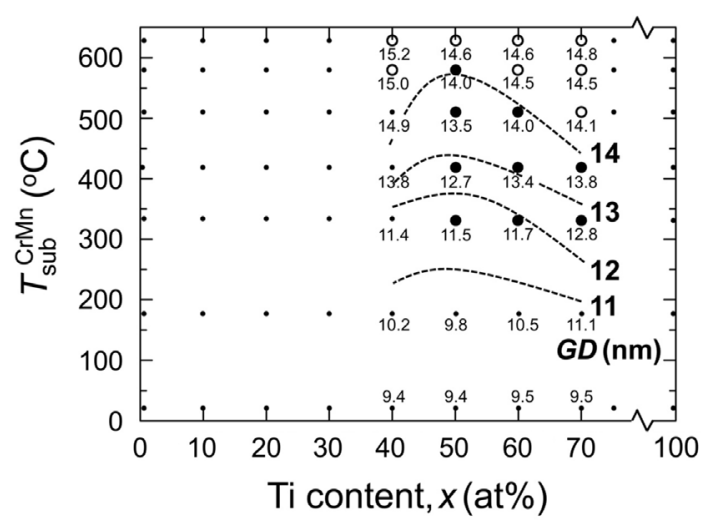

Fig. 5. Variation in grain diameter (GD) of bcc-SL with respect to substrate temperature $T_{\text {sub }}$ and Ti content $(x)$. 


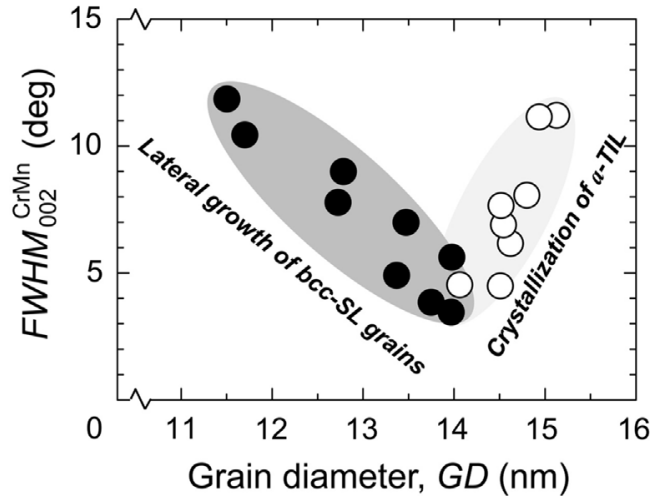

Fig. 6. Correlation between (002) FWHM and GD of bcc-SL.

(not shown in this paper). It is clearly shown that GD gradually increased as the increase of $T_{\text {sub }}$ regardless of $x$. The result indicates that the high substrate temperature enhances the adatomic mobility during solidification that induces lateral grain growth in the film.

Figure 6 represents the correlation between (002) FWHM and GD of bcc-SLs. (002) FWHM decreased from $11.8^{\circ}$ to $3.4^{\circ}$ as the increase of GD from $11.5 \mathrm{~nm}$ to $14.0 \mathrm{~nm}$, and then (002) FWHM increased as further increase of GD. While the development of the lateral growth for bcc-SL grain suppresses (002) FWHM, crystallization of the amorphous Cr-Ti TILs deteriorates the quality of (002) texture. The result suggests that exploring the amorphous materials with the high crystallization temperature is inevitable to realize highly (002) textured SL.

\subsection{Magnetic properties of FePt-C granular films} with highly (002) textured bec-SL

FePt-C granular films were prepared to clarify the effect of (002) FWHM of bcc-SL on the magnetic properties of the film. The stacked structure consists of FePt-C/MgO/CrMn/CrTi/glass sub. (002) FWHM of bccSL was manipulated by changing the fabrication conditions for $\mathrm{CrMn}$ and $\mathrm{CrTi}$ whereas the sputtering conditions for $\mathrm{MgO}$ and FePt-C was fixed. To promote the $\mathrm{L} 1_{0}$ ordered phase for $\mathrm{FePt}$, the substrate temperature was elevated about $600{ }^{\circ} \mathrm{C}$ before deposition of FePt-C film [7, 8]. Figure 7 shows the out-of-plane magnetization curve for the $\mathrm{L}_{0}$ FePt-C granular films with (002) FWHM of 3.8 (solid), 4.2 (dash), and 13.7 (dot). All hysteresis loops show the constant magnetization while an applied magnetic field decreases from 65 kOe to 5 $\mathrm{kOe}$, and then the magnetization gradually decreased with the decrease in the magnetic field intensity from $5 \mathrm{kOe}$ to $-50 \mathrm{kOe}$. It is shown that the required field to reverse the magnetization is over $50 \mathrm{kOe}$. The result indicates that

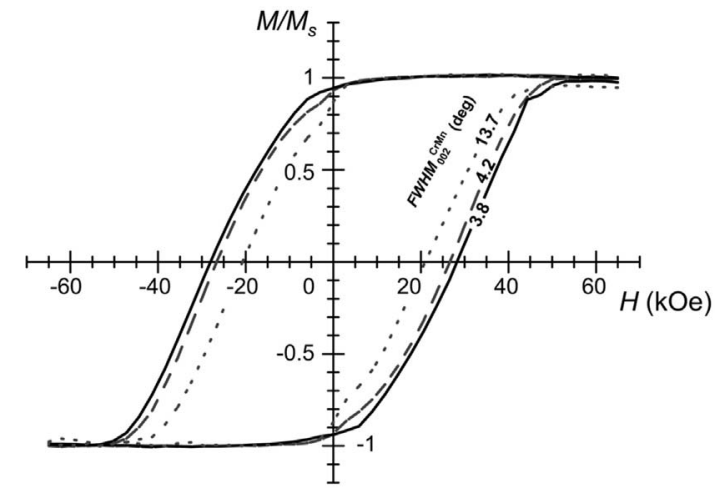

Fig. 7. Out-of-plane $\mathrm{M}-\mathrm{H}$ loops of FePt-C granular films with bcc-SL (002) FWHM of $3.8^{\circ}$ (solid line), $4.2^{\circ}$ (dashed line), and $13.7^{\circ}$ (dotted line).

the $\mathrm{L} 1_{0}$ ordered phase for FePt-C granular films in which the magnetic easy-axis is aligned toward normal to the film plane is realized. It is clear that both the coercivity and squareness $\left(M / M_{\mathrm{s}}\right)$ of the films gradually increased as the decrease in (002) FWHM. The quantitative result suggests that the reduction of (002) FWHM of bcc-SL affects to the overlying $\mathrm{MgO}$ film as well as FePt-C granular film by means of the hetero-epitaxial growth. Consequently, it is suggested that the reduction of the angular distribution of the magnetized easy axis enhances the perpendicular hysteresis.

\section{Conclusion}

$\mathrm{Cr}_{100-\mathrm{x}} \mathrm{Ti}_{\mathrm{x}}$ amorphous texture-inducing layers (TIL) were investigated to realize highly (002) textured FePt-C granular film through the (002) texture formation of CrMn SL. Asdeposited $\mathrm{Cr}_{100-\mathrm{x}} \mathrm{Ti}_{\mathrm{x}}$ TILs showed amorphous phase in the range of $30 \leq x($ at $\%) \leq 75$. Particularly, those films with the composition range of $40 \leq x \leq 60$ kept the amorphous phase against the heat treatment over $600{ }^{\circ} \mathrm{C}$. It was found that the preference of the crystallographic orientation for CrMn SL is directly affected by structural phase of $\mathrm{Cr}_{100-\mathrm{x}} \mathrm{Ti}_{\mathrm{x}}$ TILs. (002) preferred crystal orientation was realized when CrMn is deposited on amorphous $\mathrm{Cr}_{100-\mathrm{x}} \mathrm{Ti}_{\mathrm{x}}$ TILs $(40 \leq x \leq 70)$. Otherwise, (110) orientation was formed. Correlation between the angular distribution of CrMn (002) crystal orientation (FWHM) and the grain diameter (GD) of CrMn SLs has been examined. While the development of the lateral growth for CrMn grain suppress FWHM, crystallization of $\mathrm{Cr}$-Ti $a$-TILs hinders the quality of (002) texture. It is suggested that the reduction of the angular distribution of the magnetic easy axis due to the highly (002) textured CrMn SL enhances the perpendicular hysteresis in FePt-C granular film. 


\section{References}

[1] M. H. Kryder, E. C. Gage, T. W. McDaniel, W. A. Challener, R. E. Rottmayer, G. Ju, Y.-T. Hsia, and M. F. Erden, Proc. IEEE 96, 1810 (2008).

[2] A. Q. Wu, Y. Kubota, T. Klemmer, T. Rausch, C. Peng, Y. Peng, D. Karns, X. Zhu, Y. Ding, E. K. C. Chang, Y. Zhao, H. Zhou, K. Gao, J.-U. Thiele, M. Seigler, G. Ju, and E. Gage, IEEE Trans. Magn. 49, 779 (2013).

[3] X. Wang, K. Gao, H. Zhou, A. Itagi, M. Seigler, and E. Gage, IEEE Trans. Magn. 49, 686 (2013).
[4] Seong-Jae Jeon, Shintaro Hinata, Shin Saito, and Migaku Takahashi, J. Appl. Phys. 117, 17A924 (2015).

[5] Byung-Joo Lee, Metall. Trans. A 24, 1919 (1993).

[6] B. D. Cullity and S. R. Stork, Elements of X-ray Diffraction, Prentice Hall, New Jersey (2001) pp. 167-171.

[7] S. Wicht, V. Neu, L. Schultz, V. Mehta, S. Jain, J. Reiner, O. Mosendz, O. Hellwig, D. Weller, and B. Rellinghaus, J. Appl. Phys. 117, 013907 (2015).

[8] J. Wang, S. Hata, Y. K. Takahashi, H. Sepehri-Amin, B.S.D.Ch.S. Varaprasad, T. Shiroyama, T. Schreflc, and K. Hono, Acta Mater. 91, 41 (2015). 